\title{
Cardiac remodeling and subclinical left ventricular dysfunction in adults with uncomplicated obesity: a cardiovascular magnetic resonance study
}

\author{
Jing Liu ${ }^{\# \#}$, Jing $\mathrm{Li}^{2 \#}$, Huaxia $\mathrm{Pu}^{1}$, Wenzhang $\mathrm{He}^{1}$, Xiaoyue $\mathrm{Zhou}^{3}$, Nanwei Tong ${ }^{2}$, Liqing Peng ${ }^{1 \wedge}$ \\ ${ }^{1}$ Department of Radiology, West China Hospital, Sichuan University, Chengdu, China; ${ }^{2}$ Department of Endocrinology and Metabolism, West China \\ Hospital, Sichuan University, Chengdu, China; ${ }^{3}$ MR Collaboration, Siemens Healthineers Ltd., Shanghai, China
}

Contributions: (I) Conception and design: J Liu, J Li, L Peng, N Tong; (II) Administrative support: L Peng, N Tong; (III) Provision of study materials or patients: J Liu, J Li; (IV) Collection and assembly of data: J Liu, J Li, L Peng, H Pu, W He, X Zhou; (V) Data analysis and interpretation: All authors; (VI) Manuscript writing: All authors; (VII) Final approval of manuscript: All authors.

\#These authors contributed equally to this work.

Correspondence to: Liqing Peng, MD, PhD. Department of Radiology, West China Hospital, Sichuan University, 37 Guoxue Alley, Chengdu 610041, China. Email: pengliqing@wchscu.cn; Nanwei Tong, MD, PhD. Department of Endocrinology and Metabolism, West China Hospital, Sichuan University, 37 Guoxue Alley, Chengdu 610041, Sichuan, China. Email: tongnw@scu.edu.cn.

Background: Obesity often exists alongside comorbidities and increases the risk of heart failure and cardiovascular mortality. However, the specific effects of obesity on cardiac structure and function have not been clarified. This study set out to evaluate left ventricular (LV) geometric and functional changes using cardiovascular magnetic resonance imaging (CMR) in adults with uncomplicated obesity.

Methods: Forty-eight patients with uncomplicated obesity [body mass index (BMI) mean \pm SD: $29.8 \pm 2.1 \mathrm{~kg} / \mathrm{m}^{2}$ ] and 25 healthy controls were included in this study. CMR was used to assess LV geometry, global systolic function, and strains, and to quantify epicardial adipose tissue (EAT). Body composition was measured by dual X-ray absorptiometry.

Results: Compared with healthy controls, patients with obesity had increased LV size, mass, and myocardial thickness, and impaired myocardial contractility, with lower global radial, circumferential, and longitudinal peak strains (PS), and circumferential and longitudinal peak diastolic strain rates (PDSR; all $\mathrm{P}<0.05)$. Multivariable linear regression showed that BMI was independently associated with LV maximum myocardial thickness (LVMMT) $(\beta=0.197, \mathrm{P}=0.016)$. Visceral adipose tissue (VAT) was independently associated with LV global longitudinal PS $(\beta=-2.684, \mathrm{P}=0.001)$, and both longitudinal $(\beta=-0.192, \mathrm{P}=0.002)$ and circumferential $(\beta=-0.165, \mathrm{P}=0.014) \mathrm{PDSR}$. Homeostasis model assessment of insulin resistance (HOMA-IR) was mildly correlated with BMI ( $\mathrm{r}=0.327)$ and body fat percentage $(\mathrm{BF} \%)(\mathrm{r}=0.295)$ in patients with obesity (all $\mathrm{P}<0.05)$. HOMA-IR was independently associated with LV global circumferential PS $(\beta=-0.276, \mathrm{P}=0.04)$ and PDSR $(\beta=-0.036, \mathrm{P}=0.026)$.

Conclusions: Extensive LV geometric remodeling and marked changes in cardiac strains were observed in adults with obesity. Tissue tracking with CMR can reveal subclinical impaired ventricular function with preserved LV ejection fraction in such patients. BMI was independently related to LV remodeling in obesity. HOMA-IR and VAT are potentially superior to BMI as predictors of subclinical dysfunction, assessed by strain, in obesity.

\footnotetext{
^ ORCID: 0000-0002-2376-3268.
} 
Trial Registry: This study has been registered with the Chinese Clinical Trial Registry (ID: ChiCTR1900026476; Effect of lifestyle intervention on metabolism of obese patients based on smart phone software).

Keywords: Cardiac remodeling; cardiovascular magnetic resonance imaging (CMR); left ventricle; obesity; strain

Submitted Jul 13, 2021. Accepted for publication Nov 26, 2021.

doi: $10.21037 /$ qims-21-724

View this article at: https://dx.doi.org/10.21037/qims-21-724

\section{Introduction}

The global prevalence of overweight and obesity has increased markedly in recent decades. Since the beginning of the 21 st century, obesity has become an epidemic in China, with an estimated increase of $0.32 \%$ per year in young and middle-aged adults (1). Being overweight or obese increases the risk of cardiovascular disease and heart failure. One study showed that elevated body mass index (BMI) was associated with an increased risk of heart failure in the absence of a threshold (2). Obesity-related comorbidities, such as hypertension, diabetes, and coronary heart disease, are major contributors to heart failure. However, even in the absence of any clinically apparent cardiovascular risk factors, individuals with obesity exhibit notable subclinical structural and functional changes that predispose them to future heart failure (3-5).

Ejection fraction is the most widely used parameter to evaluate global ventricular systolic function. However, it may not be an optimal marker for the evaluation of cardiac dysfunction in the obese population. Kenchaiah et al. (2) found that approximately half of their obese patients with heart failure had a preserved left ventricular ejection fraction (LVEF), as assessed by echocardiography. In another study with 5,098 participants, LVEF was also found to be insensitive to myocardial changes related to obesity (6). It is worth noting that most studies to investigate the effects of obesity on cardiac structure and function have used echocardiography $(5,7,8)$. However, because acoustic windows become more limited as BMI increases, echocardiography may have reduced accuracy in patients with obesity. Furthermore, the measurement of left ventricular (LV) morphology based on Simpson's biplane method has limitations in terms of computing LV mass and volume (9). Cardiovascular magnetic resonance imaging (CMR) based on three-dimensional (3D) cine images of the heart aids in assessing ventricular geometry and function with high accuracy and reproducibility (10). Currently,
CMR tissue tracking is widely used to identify subclinical myocardial dysfunction, owing to its high sensitivity in measuring global and regional cardiac mechanics through the tracking of myocardial motion (11). Early detection of subclinical cardiac changes resulting from obesity is critical for the initiation of management to prevent the progression of cardiac failure.

The aims of this study were to use CMR to explore changes in cardiac geometry and function in individuals with obesity unaccompanied by any clinically apparent cardiovascular risk factors, and to determine the association between adiposity distribution and cardiac changes.

We present the following article in accordance with the Materials Design Analysis Reporting (MDAR) checklist (available at https://dx.doi.org/10.21037/qims-21-724).

\section{Methods}

\section{Study population}

Forty-eight individuals with obesity (defined as BMI $\geq 27.5 \mathrm{~kg} / \mathrm{m}^{2}$; range, $27.5-34.9 \mathrm{~kg} / \mathrm{m}^{2}$ ) and 25 healthy controls (HCs; BMI $<23 \mathrm{~kg} / \mathrm{m}^{2}$ ) were prospectively recruited to the study between September 2019 and February 2021. Individuals were excluded if they had any of the following: hypertension, diabetes (based on an oral glucose tolerance test), a history of cardiovascular diseases or any cardiovascular procedures, major systemic diseases that could affect the myocardium (e.g., connective tissue diseases and sarcoidosis), endocrine diseases (e.g., hyperthyroidism and hypothyroidism), metabolic diseases (e.g., a history of alcohol abuse and amyloidosis), obstructive sleep apnea, and/or any contraindication to CMR.

This study complied with the Declaration of Helsinki (as revised in 2013) and was approved by the Institutional Review Board of the West China Hospital in Sichuan University. Written informed consent was obtained from all study participants. 


\section{Baseline data collection}

Baseline data were collected for all participants and included a medical history, anthropometric measurements (weight and height), heart rate, and blood pressure (BP). $\mathrm{BP}$ was measured twice by an experienced researcher using an automatic oscillometric cuff sphygmomanometer in the sitting position after 15 minutes' rest. The mean of two consecutive readings was calculated. Heart rate was assessed using a 12-lead electrocardiogram (ECG) and recorded on a Vitaport system (Temec Instruments BV, Kerkrade, the Netherlands) at $256 \mathrm{~Hz}$ at rest. Fasting blood glucose (FBG) and serum lipid profiles, including total cholesterol, triglycerides, high-density lipoprotein (HDL), and lowdensity lipoprotein (LDL), were also measured.

Mean arterial pressure (MAP; $\mathrm{mmHg}$ ) was calculated as follows: $\mathrm{MAP}=(\mathrm{SBP}+2 \mathrm{DBP}) / 3$, where SBP and DBP are systolic and diastolic BP, respectively. Fasting insulin (FINS) was measured in 45 participants with obesity.

Homeostasis model assessment of insulin resistance (HOMA-IR) was calculated as follows: HOMA-IR $=[$ FBG $(\mathrm{mmol} / \mathrm{L}) \times \mathrm{FINS}(\mathrm{mU} / \mathrm{L})] / 22.5$.

\section{Assessment of obesity}

Waist circumference (WC) was measured midway between the last rib and the iliac crest, and hip circumference was measured at the largest diameter of the hip. WC and hip circumference were measured to the nearest $0.5 \mathrm{~cm}$. The waist-to-hip ratio (WHR) and the waist-to-height ratio (WHtR) were calculated. WC, WHR, and WHtR are indices predictive of central obesity. BMI was calculated as weight $(\mathrm{kg})$ divided by height squared $\left(\mathrm{m}^{2}\right)$. According to the Chinese criteria (1), BMI was categorized into three groups: healthy weight (BMI $18.5-23.0 \mathrm{~kg} / \mathrm{m}^{2}$ ), overweight (BMI $23.0-27.5 \mathrm{~kg} / \mathrm{m}^{2}$ ), and obese (BMI $\geq 27.5 \mathrm{~kg} / \mathrm{m}^{2}$ ). Total fat, visceral adipose tissue (VAT), and body fat percentage (BF\%) were measured by dual X-ray absorptiometry (Lunar iDXA; GE Medical Systems Lunar, Madison, WI, USA). Body surface area was calculated using the Du Bois formula (12).

\section{CMR protocol}

Participants underwent CMR examination in a supine position. CMR images were acquired using a 3-T wholebody scanner (MAGNETOM Skyra; Siemens Healthcare,
Erlangen, Germany) with an 18-channel phased-array body coil. With a standard ECG-triggering device, data were acquired during the end-inspiratory breath-hold period. A segmented breath-hold balanced steady-state free precession (bSSFP) sequence was used to obtain 8-14 continuous cine images from the base to the apex in the short-axis view, and LV two- and four-chamber cine images in the long-axis view. The bSSFP parameters were as follows: TR/TE 3.3/1.22 ms; flip angle $41^{\circ}$; slice thickness $8 \mathrm{~mm}$; field of view $360 \mathrm{~mm} \times 320 \mathrm{~mm}$; and matrix size $208 \times 166$. Contrast medium (gadobenate dimeglumine; MultiHance; $0.5 \mathrm{mmol} / \mathrm{mL}$; Bracco, Milan, Italy) was injected into the right antecubital vein with a power injector (Stellant; MEDRAD) at a flow rate of $3.0 \mathrm{~mL} / \mathrm{s}$, which was followed by injection of $20 \mathrm{~mL}$ saline. Late gadolinium enhancement (LGE) images were obtained after contrast injection using a segmented phase-sensitive inversion recovery (PSIR) turbo FLASH sequence over a mean duration of 10-15 minutes. The PSIR sequence parameters were as follows: TR/TE 3.0/1.24 ms; flip angle $40^{\circ}$; slice thickness $8 \mathrm{~mm}$; field of view $340 \mathrm{~mm} \times 284 \mathrm{~mm}$; and matrix size $256 \times 184$.

\section{CMR image analysis}

All CMR data were imported into a commercially available software program (cvi42 version 5.11.3; Circle Cardiovascular Imaging, Calgary, Canada). Two radiologists with more than 3 years of experience (JL and LQP) analyzed the measurements while blinded to participant status (i.e., obesity $v s$. control group).

\section{Quantification of epicardial adipose tissue (EAT)}

EAT is represented by regions of high signal intensity between the myoepicardium and parietal pericardium. To acquire quantitative EAT, short-axis and long-axis four-chamber cine slices were imported into the tissue char module in cvi 42. EAT volume was measured on the short-axis cine slices during the end-diastolic phase. The myoepicardial and parietal pericardial contours were manually delineated per slice, extending from the mitral valve hinge down to the ventricular apex. The EAT area was measured on the single four-chamber view cine images during the end-diastolic phase. Regions of high signal intensity between the myoepicardium and parietal pericardium were semi-automatically traced and calculated after the exclusion of blood vessels (Figure 1A-1C). 

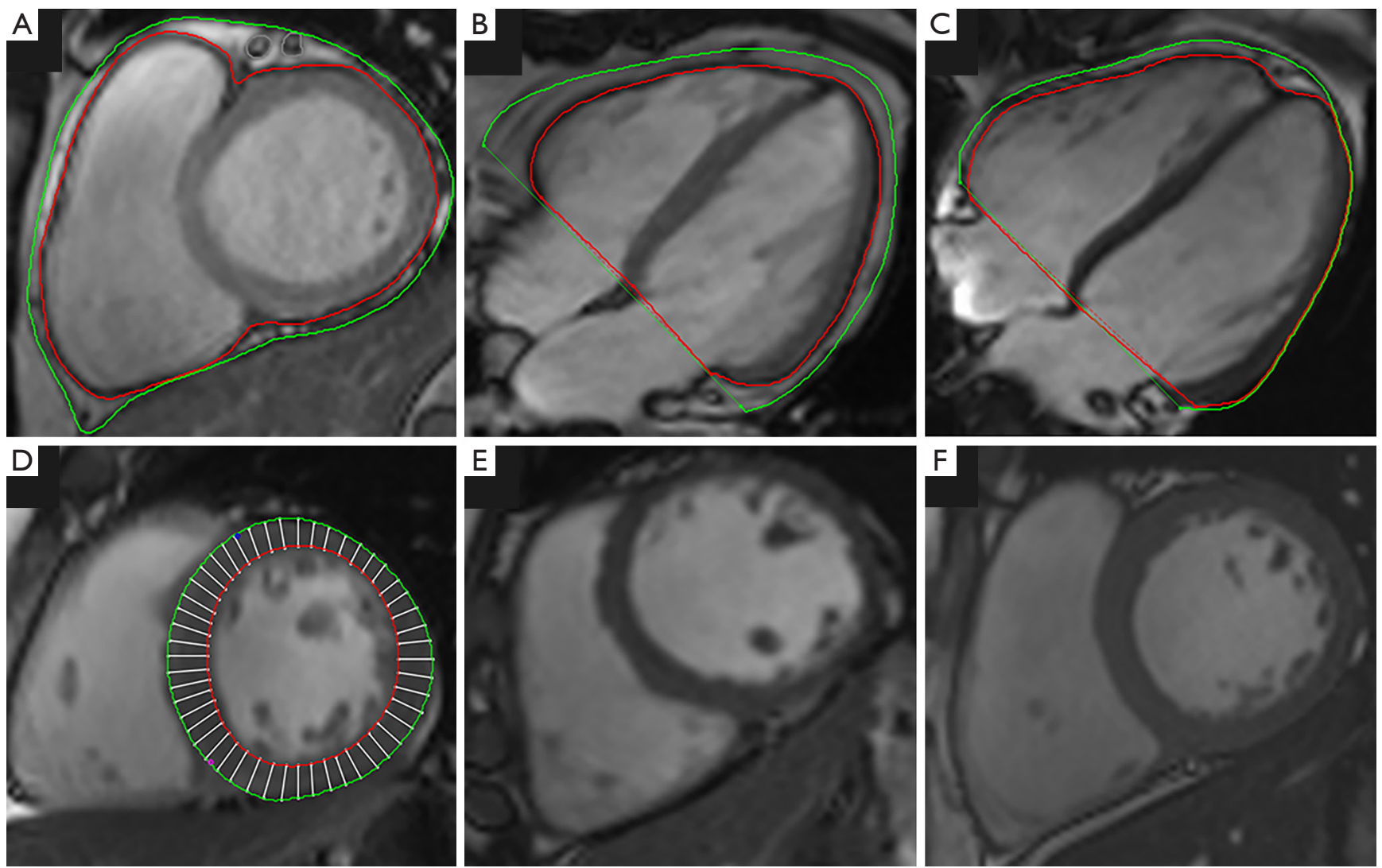

Figure 1 Quantification of epicardial adipose tissue (EAT) and left ventricular (LV) myocardial thickness. The EAT area is delineated on (A) a short-axis steady-state free precession (SSFP) image with blood vessels excluded, (B) a four-chamber view in an individual with obesity, and (C) in a normal-weight individual. (D) Three-dimensional LV thickness was acquired using short-axis SSFP images. (E,F) Representative images of myocardial thickness in healthy $(\mathrm{E})$ and obese $(\mathrm{F})$ individuals.

\section{Geometry and global systolic function}

The endo- and epicardial contours of the LV myocardium on the short-axis cine images were manually traced during the end-diastolic and end-systolic phases of the cvi42 short-3D module. Parameters of LV global systolic function and geometry, namely LVEF, LV enddiastolic volume (LVEDV), LV end-systolic volume (LVESV), stroke volume (SV), cardiac output (CO), and LV mass at end-diastole, were computed automatically. $\mathrm{LV}$ mass was indexed to height ${ }^{2.7}[\mathrm{LV}$ mass index (LVMI); $\left.\mathrm{g} / \mathrm{m}^{2.7}\right]$ (13). Concentricity was calculated as the ratio of $\mathrm{LV}$ mass to $\mathrm{LVEDV}(\mathrm{g} / \mathrm{mL})$. Mean $\mathrm{LV}$ regional values for 16 myocardial segment thicknesses (excluding the apex) were also automatically computed [American Heart Association standard 17-segment model (14); Figure $1 D-1 F]$. Finally, the LV maximum myocardial thickness (LVMMT) was calculated.

\section{Myocardial strains}

The long-axis two- and four-chamber and short-axis cine slices were uploaded into the $3 \mathrm{D}$ tissue-tracking module for LV myocardial strain analysis. The endo- and epicardial contours were manually delineated per slice during the end-diastolic phase in all series, with the papillary muscles and moderator bands excluded. LV global myocardial strain parameters were acquired automatically, and included the radial, circumferential, and longitudinal peak strains (PS), peak systolic strain rates (PSSR), and peak diastolic strain rates (PDSR). LV strain is defined as the degree of myocardial deformation from the initial length of the myocardium $\left(\mathrm{L}_{0}\right.$; in end-diastole) to its maximum length ( $\mathrm{L}$; in end-systole), and is calculated using the following formula: Myocardial strain $=\left(\mathrm{L}-\mathrm{L}_{0}\right) / \mathrm{L}_{0}$. Strain rate represents the rate of shortening of length (15). Longitudinal, circumferential, and radial strain and strain 
rate can be calculated for different dimensions of myocardial deformation.

\section{Reproducibility}

Intra- and interobserver variability in indices of EAT, LV geometry, traditional function, and myocardial strain were analyzed in 20 randomly selected participants (obesity group, $\mathrm{n}=12$; HCs, $\mathrm{n}=8$ ). To determine intraobserver variability, one radiologist (JL) measured the same image twice within a period of 1 month. To evaluate interobserver variability, a second radiologist (HXP), who was blinded to the measurements made by the first observer, reanalyzed the measurements.

\section{Statistical analysis}

All statistical analyses were performed with SPSS version 23 (IBM, Armonk, NY, USA). Normally distributed continuous data from the obesity and HC groups were expressed as the mean \pm SD and compared using Student's $t$-tests. Binary variables were analyzed using crosstab chisquare tests. Pearson's correlation coefficient was used to determine correlations between adiposity (estimated by $\mathrm{BMI}$, total fat, VAT, BF\%, EAT volume, EAT area, WC, WHR, and WHtR), insulin resistance, and parameters of LV geometry, function, and strain. Correlations between cardiac geometry and BP, as well as relationships between cardiac geometry, function, and ventricular strain were also assessed in patients with obesity. Multivariable linear regression was used to estimate the association between insulin resistance and adiposity (BMI, BF\%, VAT, WC, and WHR) and LV geometry, global systolic function, and strain. Adjustments were made to the basic unadjusted model. Model 1 included age and sex for BMI, and age, sex, and height for insulin resistance, $\mathrm{BF} \%, \mathrm{VAT}, \mathrm{WC}$, and $\mathrm{WHR}$. In Model 2, to determine the association between obesity and cardiac remodeling and function independent of BP, MAP was added to the outcomes to adjust for BP. HOMA-IR was also added to assess the association of adiposity with cardiac remodeling and function. Intraclass correlation coefficients (ICCs) were used to evaluate both inter- and intraobserver variability. Two-sided $\mathrm{P}<0.05$ was considered statistically significant.

\section{Results}

\section{Baseline characteristics}

This study included 48 individuals with obesity (BMI
$29.8 \pm 2.1 \mathrm{~kg} / \mathrm{m}^{2}$ ) and $25 \mathrm{HCs}\left(\mathrm{BMI} 21.2 \pm 1.3 \mathrm{~kg} / \mathrm{m}^{2}\right.$ ), the baseline characteristics of whom are presented in Table 1 . The mean age, height, proportion of males, FBG, serum lipid profiles, and heart rate were comparable between the two groups. Compared with the HCs, participants with obesity had higher BP, but the BP values were still within the normal range. Furthermore, the obesity group had greater adiposity (EAT volume and area, total fat, $\mathrm{BF} \%$, VAT, WC, hip circumference, WHR, and WHtR) than the $\mathrm{HC}$ group.

\section{Comparison of CMR findings between the obesity and $\mathrm{HC}$ groups}

Parameters of LV geometry (LVEDV, LVESV, LV mass, LVMI, and LVMMT) were greater, but the LV mass/volume ratio was similar, in the obesity group compared with the HC group (Table 2). With regard to global systolic function, individuals with obesity had an increased SV (98.6 \pm 18.5 vs. 83.6 $\pm 15.2 \mathrm{~mL} ; \mathrm{P}<0.001)$ and $\mathrm{CO}(7.3 \pm 1.8$ vs. $6.1 \pm 1.4 \mathrm{~L} / \mathrm{min}$; $\mathrm{P}<0.001)$, but similar LVEF $(62.6 \% \pm 4.6 \%$ vs. $62.3 \% \pm 4.5 \%$, $\mathrm{P}=0.779)$, compared with the HCs. Regarding LV strain, the obesity group had lower global PS in three spatial directions and circumferential and longitudinal PDSRs than the HC group (Figure 2).

\section{Associations between cardiac geometry and BP in obese individuals}

In the obesity group, MAP was positively correlated with LVEDV, LVESV, LV mass, and LVMMT, but not with LVMI or the LV mass/volume ratio (Table 3).

\section{Associations between cardiac geometry, function, and ventricular strain in individuals with obesity}

In individuals with obesity, there was a weak positive correlation between LVEF and radial PS ( $r=0.297$; $=0.041)$, whereas negative correlations were observed between radial PS and LVEDV, LVESV, and LV mass. Further, LVMMT and LV mass were negatively correlated with longitudinal PS. No cardiac geometric or functional parameters were correlated with circumferential PS (Table 4).

\section{Association between HOMA-IR and adiposity in individuals with obesity}

Pearson's correlation was used to evaluate associations 
Table 1 Baseline characteristics of the study cohort

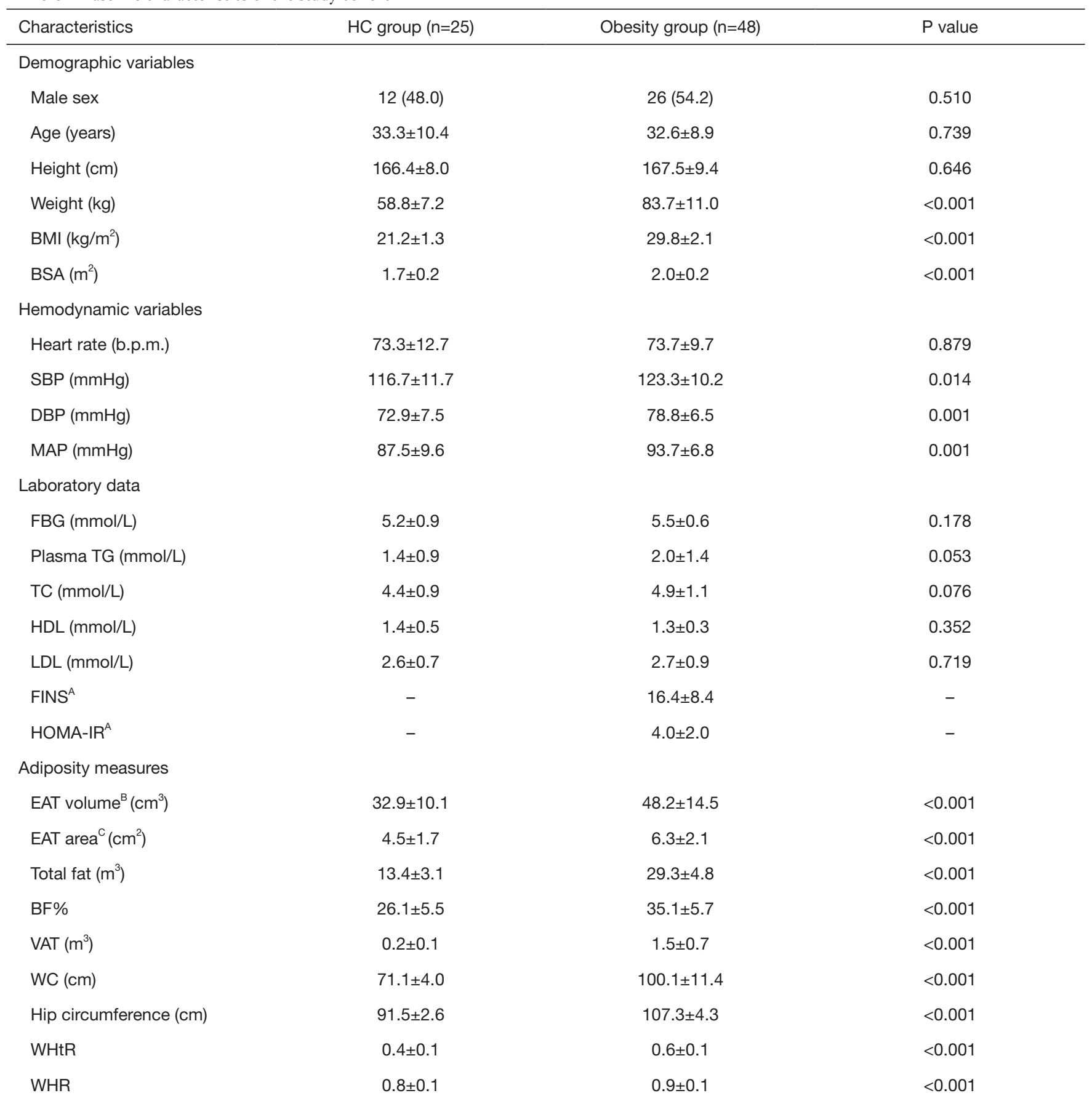

Unless indicated otherwise, data are given as mean \pm SD or $n(\%) .{ }^{A}$, fasting insulin (FINS) was not measured in 3 of the 48 participants in the obesity group and in none of the healthy controls (HCs); consequently, these individuals were not included in homeostasis model assessment of insulin resistance (HOMA-IR) analysis. ${ }^{\text {B }}$, epicardial adipose tissue (EAT) volume was obtained from short-axis cine slices. ${ }^{\mathrm{C}}$, EAT area was obtained from a single long-axis four-chamber cine slice. BF\%, body fat percentage; BMI, body mass index; BSA, body surface area; DBP, diastolic blood pressure; FBG, fasting plasma glucose; HDL, high-density lipoprotein; LDL, low-density lipoprotein; MAP, mean arterial pressure; SBP, systolic blood pressure; TC, total cholesterol; TG, triglycerides; VAT, visceral adipose tissue; WC, waist circumference; WHR, waist-to-hip ratio; WHtR, waist-to-height ratio. 
Table 2 Cardiac geometry and functional and strain parameters in the obesity and healthy control groups

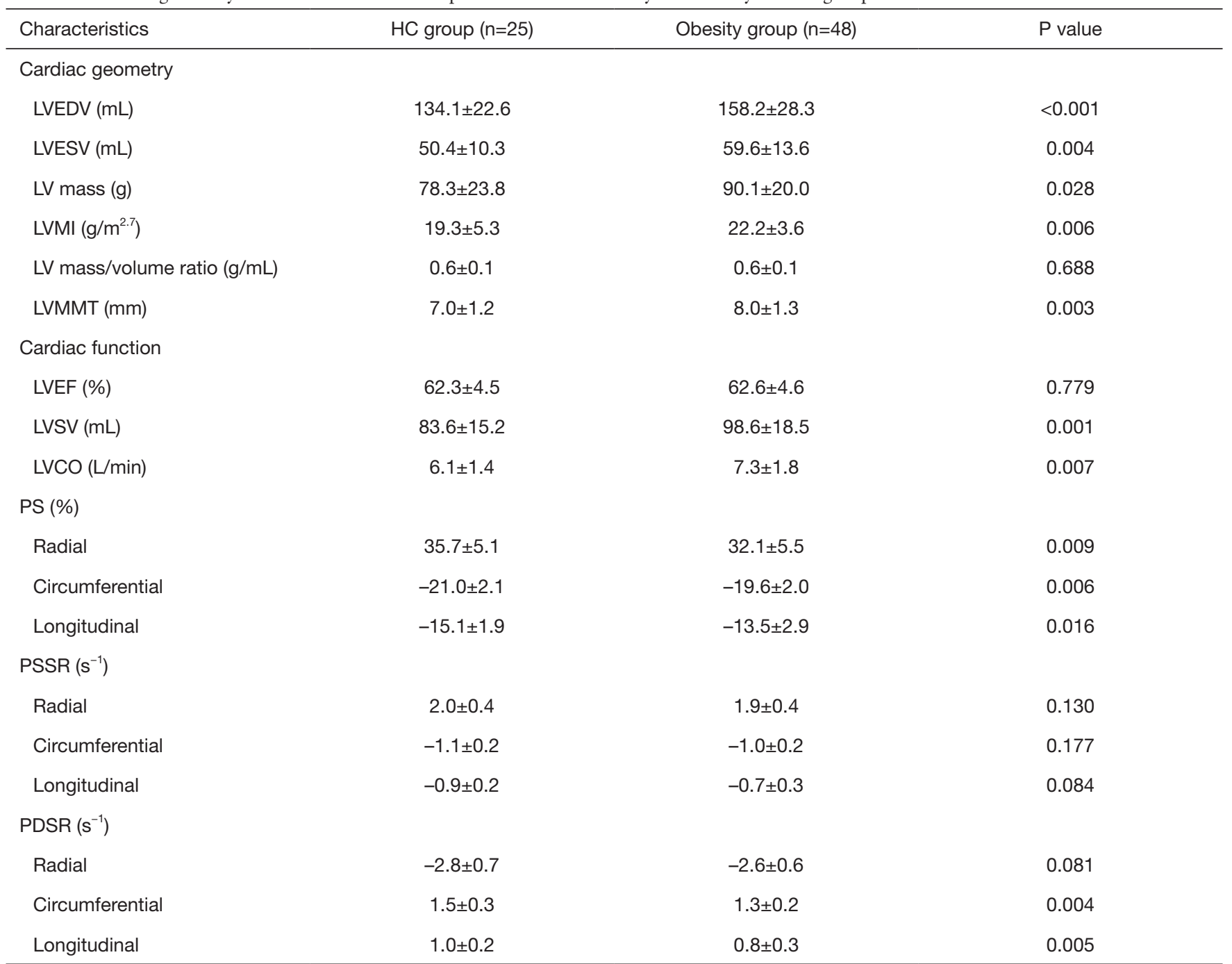

Unless indicated otherwise, data are given as mean \pm SD. HC, healthy control; LV, left ventricle; LVCO, LV cardiac output; LVEDV, LV end-diastolic volume; LVEF, LV ejection fraction; LVESV, LV end-systolic volume; LVMI, LV mass index; LVMMT, LV maximum myocardial thickness; LVSV, LV stroke volume; PDSR, peak diastolic strain rate; PS, peak strain; PSSR, peak systolic strain rate.

between HOMA-IR and indices of adiposity (BMI, total fat, VAT, BF\%, EAT volume, EAT area, WC, WHR, and WHtR) in 45 participants with obesity. HOMA-IR was only mildly correlated with $\mathrm{BMI}(\mathrm{r}=0.327, \mathrm{P}<0.05)$ and $\mathrm{BF} \%$ $(\mathrm{r}=0.295, \mathrm{P}<0.05)$, and shared no significant correlations with any of the other indices of adiposity.

\section{Associations between HOMA-IR and LV geometry, function, and strain in individuals with obesity}

Of all the LV geometry, function, and strain parameters, only HOMA-IR was inversely associated with circumferential PS
$(\mathrm{P}<0.05)$ in the unadjusted model. Multivariate regression analysis showed that, even after adjusting for age, sex, height, and MAP (Model 2), HOMA-IR was inversely associated with both circumferential PS ( $\beta=-0.276$; $95 \%$ CI: -0.540 to $-0.013 ; \mathrm{P}=0.04)$ and circumferential PDSR $(\beta=-0.036 ; 95 \%$ CI: -0.068 to $-0.005 ; \mathrm{P}=0.026$; Table 5).

\section{Associations between adiposity and LV geometry, function, and strain in individuals with obesity}

Pearson's correlation showed that BMI was positively correlated with LVMMT and the LV mass/volume ratio 
A

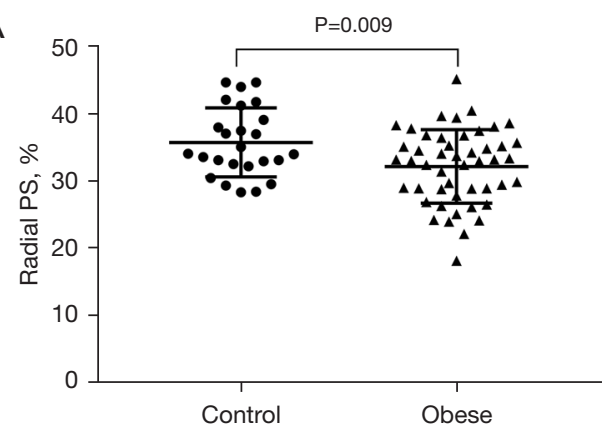

$\mathrm{C}$

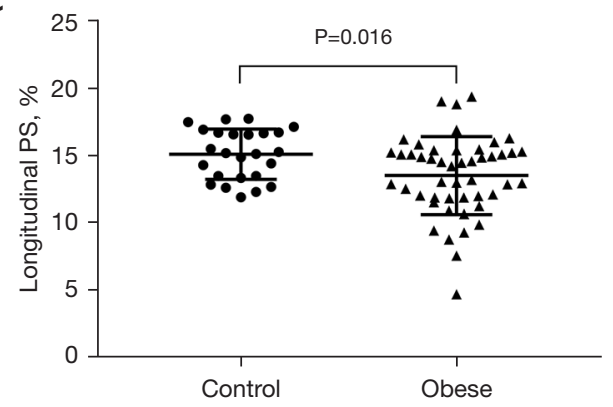

$\mathrm{E}$

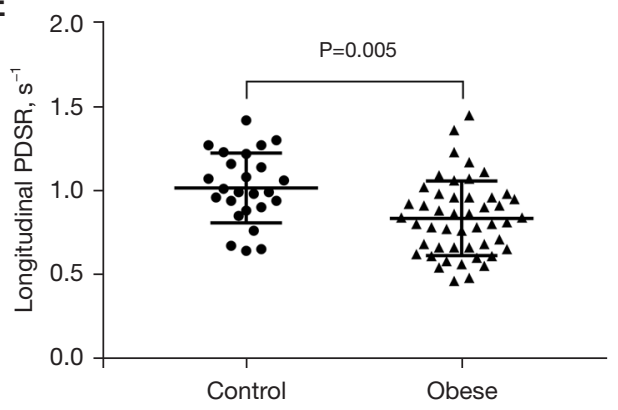

B

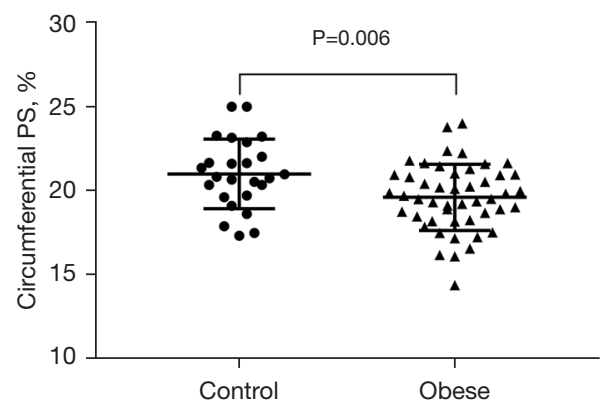

$\mathrm{D}$

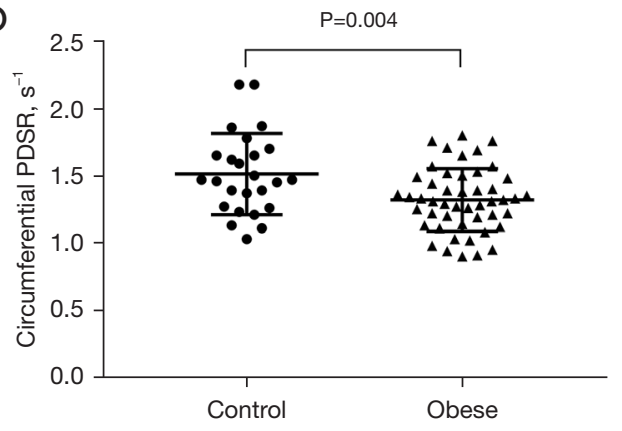

Figure 2 Strain and strain rates in individuals with obesity and healthy controls. (A) Global radial, (B) circumferential, and (C) longitudinal peak strain (PS). (D) Circumferential and (E) longitudinal peak diastolic strain rate (PDSR).

Table 3 Association (correlation coefficients) between cardiac geometry and mean arterial pressure in adults with obesity

\begin{tabular}{|c|c|c|c|c|c|c|}
\hline & LVEDV & LVESV & LV mass & LVMI & LV mass/volume ratio & LVMMT \\
\hline
\end{tabular}

*, P<0.05; ** $\mathrm{P}<0.01$. LV, left ventricle; LVEDV, LV end-diastolic volume; LVESV, LV end-systolic volume; LVMI, LV mass index (end-diastolic mass indexed to height ${ }^{2.7}$ ); LVMMT, LV maximum myocardial thickness; MAP, mean arterial pressure.

in the obesity group. In contrast, $\mathrm{BF} \%$ was negatively correlated with parameters of LV geometry (LV mass, LV mass/volume ratio, LVMMT, LVEDV, and LVESV) and SV. VAT was positively correlated with LV mass, the LV mass/ volume ratio, and LVMMT, but negatively correlated with longitudinal PS, longitudinal PDSR, and circumferential PDSR. Furthermore, WC was positively correlated with LVMMT. The WHR showed positive correlations with LVMMT and the LV mass/volume ratio (Table 6).

Regression analysis demonstrated that BMI was positively 
Table 4 Association (correlation coefficients) between cardiac geometry, function, and strains in adults with obesity

\begin{tabular}{lccc}
\hline & Radial PS & Circumferential PS & Longitudinal PS \\
\hline LVEF & $0.297^{\star}$ & 0.216 & 0.140 \\
LVEDV & $-0.375^{\star *}$ & -0.150 & -0.150 \\
LVESV & $-0.463^{\star *}$ & -0.247 & -0.202 \\
LVSV & -0.233 & -0.047 & -0.081 \\
LVCO & 0.060 & 0.130 & -0.069 \\
LVMMT & -0.209 & -0.138 & $-0.294^{*}$ \\
LV mass & $-0.342^{*}$ & -0.173 & $-0.308^{\star}$ \\
LVMI & 0.109 & -0.037 & 0.012 \\
LV mass/volume ratio & -0.063 & -0.072 & -0.269 \\
\hline
\end{tabular}

*, P<0.05; **, P<0.01. LV, left ventricle; LVCO, LV cardiac output; LVEDV, LV end-diastolic volume; LVEF, LV ejection fraction; LVESV, LV end-systolic volume; LVMI, LV mass index; LVMMT, LV maximum myocardial thickness; LVSV, LV stroke volume; PS, peak strain.

Table 5 Association between HOMA-IR and left ventricular strains in adults with obesity ( $\mathrm{n}=45$ ) using multivariable linear regression

\begin{tabular}{|c|c|c|c|c|c|c|}
\hline HOMA-IR & \multicolumn{3}{|c|}{ Circumferential PS } & \multicolumn{3}{|c|}{ Circumferential PDSR } \\
\hline Crude model & -0.282 & $-0.537,-0.027$ & 0.031 & -0.028 & $-0.059,0.002$ & 0.066 \\
\hline Model 1 & -0.280 & $-0.537,-0.024$ & 0.033 & -0.039 & $-0.070,-0.008$ & 0.016 \\
\hline Model 2 & -0.276 & $-0.540,-0.013$ & 0.04 & -0.036 & $-0.068,-0.005$ & 0.026 \\
\hline
\end{tabular}

Model 1 was adjusted for age, sex and height. Model 2 was adjusted for Model 1 plus mean arterial pressure. HOMA-IR, homeostasis model assessment of insulin resistance; $\mathrm{Cl}$, confidence interval; PDSR, peak diastolic strain rate; PS, peak strain.

correlated with LVMMT and the LV mass/volume ratio in the unadjusted model. This association persisted even after adjusting for age and sex (Model 1; LVMMT, $\beta=0.2$, 95\% CI: $0.066-0.344, \mathrm{P}=0.004 ; \mathrm{LV}$ mass/volume ratio, $\beta=0.013,95 \%$ CI: $0.003-0.024, P=0.015)$. After adjustment for age, sex, MAP, and HOMA-IR, only the relationship between BMI and LVMMT remained significant (Model 2; $\beta=0.197 ; 95 \%$ CI: 0.039-0.355; $\mathrm{P}=0.016$; Figure $3 A$ ). BF\%, VAT, WC, and WHR were not significantly associated with parameters of cardiac remodeling after adjustment for different variables in Model 1 or Model 2 (Table 7).

Regression analysis revealed that, after adjusting for age, sex, height, MAP, and HOMA-IR (Model 2), VAT was independently associated with $\mathrm{LV}$ global longitudinal PS ( $\beta=-2.684 ; 95 \%$ CI: -4.084 to $-1.213 ; \mathrm{P}=0.001)$, longitudinal PDSR ( $\beta=-0.192$; 95\% CI: -0.311 to -0.074 ; $\mathrm{P}=0.002)$, and circumferential PDSR $(\beta=-0.165 ; 95 \% \mathrm{CI}$ : -0.293 to $-0.036 ; \mathrm{P}=0.014$; Table 8 ; Figure $3 B-3 D$ ).

\section{Intra- and interobserver variability}

Intra- and interobserver variability for EAT quantification, cardiac geometry, function, and strain is summarized in Table 9. The ICCs for intra- and interobserver variability were 0.883 and 0.790 , respectively, for EAT volume, and 0.945 and 0.929 , respectively, for EAT area. Good intraand interobserver agreement was observed for cardiac geometry measurements, global systolic function (ICC $0.919-0.997$ and $0.843-0.989$, respectively) and LV strains (ICC $0.750-0.942$ and $0.769-0.919$, respectively).

\section{Discussion}

In the present study, we compared LV geometric and functional characteristics, determined using CMR, between individuals with obesity in the absence of clinically apparent cardiovascular risk factors and HCs. Although their LVEF 
Table 6 Association (correlation coefficients) between adiposity distribution and left ventricular geometry, function, and strain in adults with obesity

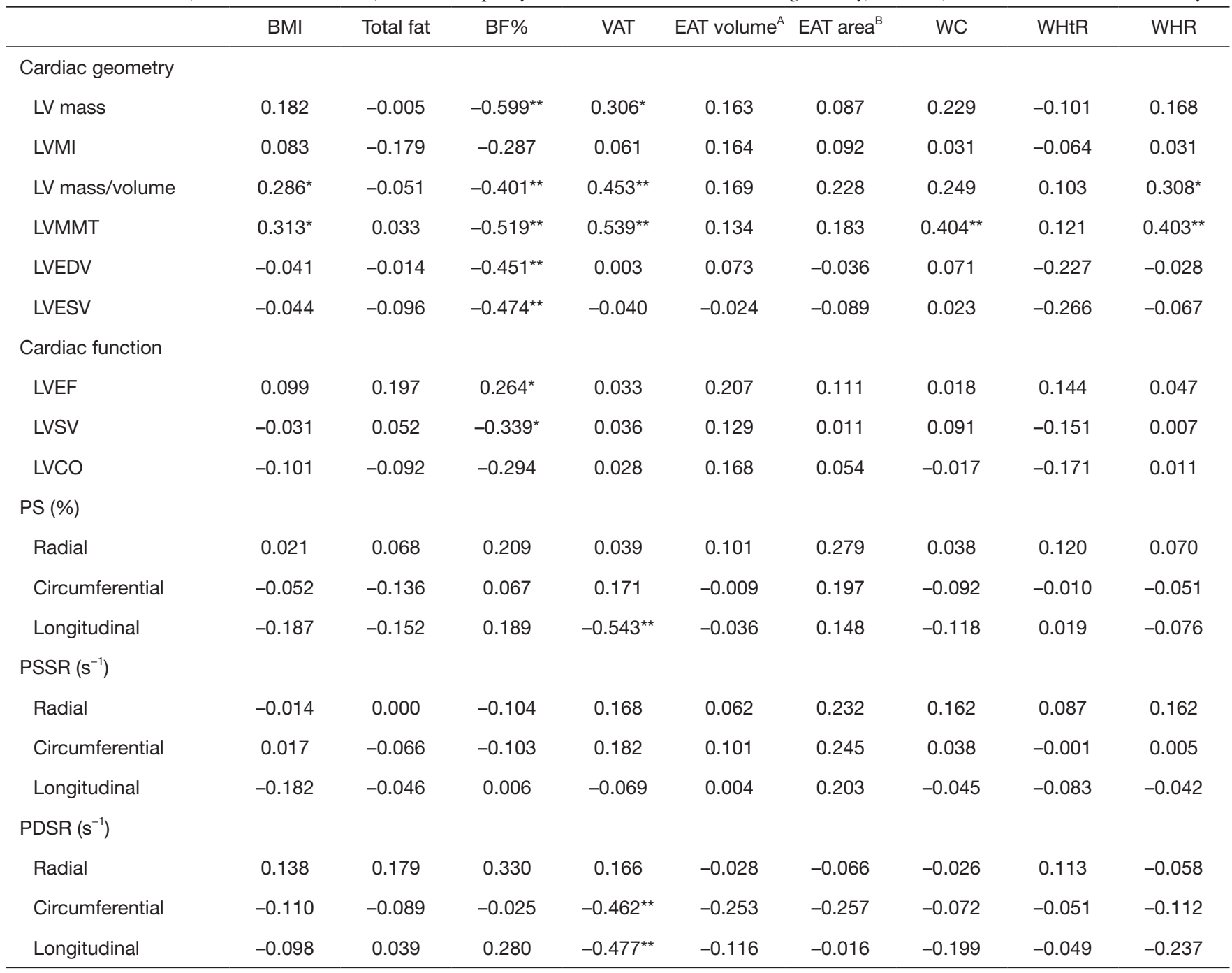

${ }^{*}, \mathrm{P}<0.05 ;{ }^{* *}, \mathrm{P}<0.01 .{ }^{\mathrm{A}}$, epicardial adipose tissue (EAT) volume was obtained from short-axis cine slices; ${ }^{\mathrm{B}}$, EAT area was obtained from a single long-axis four-chamber cine slice. BF\%, body fat percentage; BMI, body mass index; LV, left ventricle; LVCO, LV cardiac output; LVEDV, LV end-diastolic volume; LVEF, LV ejection fraction; LVESV, LV end-systolic volume; LVMI, LV mass index; LVMMT, LV maximum myocardial thickness; LVSV, LV stroke volume; PDSR, peak diastolic strain rate; PS, peak strain; PSSR, peak systolic strain rate; VAT, visceral adipose tissue; WC, waist circumference; WHR, waist-to-hip ratio; WHtR, waist-to-height ratio.

was within the normal range, compared to the HCs, participants in the obesity group had impaired myocardial contractility, with diminished PS and PDSR, and myocardial remodeling, as evidenced by increased LV size, myocardial mass, and thickness. Adiposity was correlated with cardiac geometry and functional measurements in individuals with obesity, with BMI independently related to LV remodeling and VAT independently correlated with strain assessment in adjusted models. VAT was potentially a better predictor of subclinical dysfunction than BMI in individuals with obesity.
Further, HOMA-IR was found to be inversely correlated with circumferential PS and PDSR in the obesity group, even after adjustment for age, sex, height, and MAP. This study has demonstrated the deleterious effects of obesity on myocardial geometry and subclinical LV dysfunction.

\section{$L V$ remodeling in obesity}

Cardiac remodeling is defined as molecular, cellular, and interstitial changes resulting from genomic expression, as 

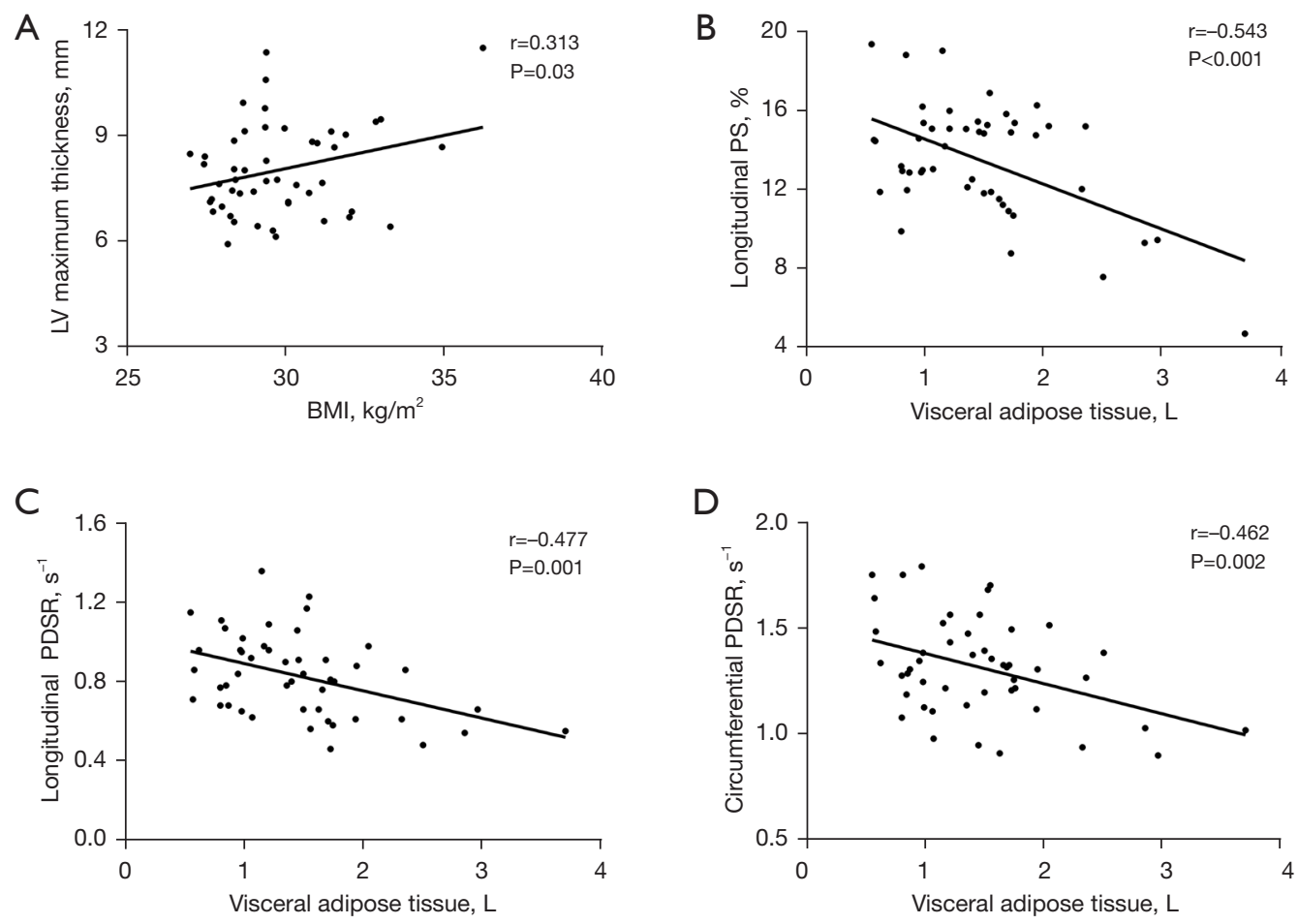

Figure 3 Association between adipose distribution and left ventricular (LV) cardiac geometry and strain in the obesity group. (A) Body mass index (BMI) was correlated with LV maximum thickness. (B-D) Visceral adipose tissue (VAT) was correlated with longitudinal peak strain (PS; B), longitudinal peak diastolic strain rate (PDSR; C), and circumferential PDSR (D).

Table 7 Association between body mass index and cardiac geometry in adults with obesity using multivariable linear regression

\begin{tabular}{|c|c|c|c|c|c|c|}
\hline BMI & \multicolumn{3}{|c|}{ LVMMT } & \multicolumn{3}{|c|}{ LV mass/volume ratio } \\
\hline Crude & 0.202 & $0.020-0.384$ & 0.030 & 0.013 & $0.000-0.025$ & 0.049 \\
\hline Model 1 & 0.200 & $0.066-0.334$ & 0.004 & 0.013 & $0.003-0.024$ & 0.015 \\
\hline Model 2 & 0.197 & $0.039-0.355$ & 0.016 & 0.012 & $-0.001-0.025$ & 0.072 \\
\hline
\end{tabular}

Model 1 was adjusted for age and sex. Model 2 was adjusted for Model 1 plus mean arterial pressure and HOMA-IR. BMI, body mass index; Cl, confidence interval; LV, left ventricle; LVMMT, LV maximum myocardial thickness; HOMA-IR, homeostasis model assessment of insulin resistance.

well as clinical manifestations of changes in size, shape, and heart function from cardiac load or injury (16). In the present study, the chamber size (LVEDV, LVESV), myocardial mass, and wall thickness were greater in the obesity group than the HC control group, which is consistent with the results of previous echocardiography and CMR studies (3,17-19). However, one study that used echocardiography reported that LVMI and chamber size did not differ significantly between an uncomplicated obesity group and a lean group (8). Accurate LV geometry evaluations using echocardiography are limited due to their dependence on operator experience, minimal acoustic windows, and low spatial resolutions. Furthermore, the geometric assumptions based on Simpson's method are compromised, especially under conditions of deformed ventricular dimensions and geometries (9). CMR is a $3 \mathrm{D}$ imaging modality with superior reproducibility and better imaging quality compared to echocardiography. Therefore, it is the preferred technique for evaluating parameters of ventricular geometry, particularly in non- 
Table 8 Multivariable association between visceral adipose tissue and left ventricular strains in adults with obesity

\begin{tabular}{|c|c|c|c|c|c|c|c|c|c|}
\hline VAT & \multicolumn{3}{|c|}{ Longitudinal PS } & \multicolumn{3}{|c|}{ Longitudinal PDSR } & \multicolumn{3}{|c|}{ Circumferential PDSR } \\
\hline Crude & -2.371 & $-3.513,-1.229$ & $<0.001$ & -0.164 & $-0.255,-0.074$ & 0.001 & -0.157 & $-0.251,-0.063$ & 0.002 \\
\hline Model 1 & -2.887 & $-4.157,-1.167$ & $<0.001$ & -0.185 & $-0.290,-0.080$ & 0.001 & -0.198 & $-0.315,-0.080$ & 0.002 \\
\hline Model 2 & -2.684 & $-4.084,-1.213$ & 0.001 & -0.192 & $-0.311,-0.074$ & 0.002 & -0.165 & $-0.293,-0.036$ & 0.014 \\
\hline
\end{tabular}

Model 1 was adjusted for age, sex, and height. Model 2 was adjusted for Model 1 plus mean arterial pressure and HOMA-IR. CI, confidence interval; PDSR, peak diastolic strain rate; PS, peak strain; HOMA-IR, homeostasis model assessment of insulin resistance.

Table 9 Comparison of inter- and intraobserver variability for epicardial adipose tissue, cardiac geometry, and peak strains

\begin{tabular}{|c|c|c|c|c|}
\hline Characteristics & \multicolumn{2}{|c|}{ Intraobserver variability $(\mathrm{n}=20)$} & \multicolumn{2}{|c|}{ Interobserver variability $(n=20)$} \\
\hline \multicolumn{5}{|c|}{ Epicardial adipose tissue } \\
\hline Volume & 0.883 & $0.705-0.957$ & 0.790 & $0.577-0.923$ \\
\hline Area & 0.945 & $0.919-0.965$ & 0.929 & $0.859-0.955$ \\
\hline LV mass & 0.988 & $0.964-0.996$ & 0.949 & $0.857-0.982$ \\
\hline LVMMT & 0.997 & $0.993-0.999$ & 0.989 & $0.970-0.996$ \\
\hline LVEF & 0.919 & $0.741-0.973$ & 0.843 & $0.588-0.943$ \\
\hline \multicolumn{5}{|l|}{ PS (\%) } \\
\hline Longitudinal & 0.851 & $0.627-0.945$ & 0.819 & $0.555-0.933$ \\
\hline \multicolumn{5}{|l|}{$\operatorname{PSSR}\left(\mathrm{s}^{-1}\right)$} \\
\hline Radial & 0.889 & $0.711-0.960$ & 0.774 & $0.474-0.914$ \\
\hline Circumferential & 0.917 & $0.779-0.970$ & 0.919 & $0.784-0.971$ \\
\hline Longitudinal & 0.750 & $0.553-0.849$ & 0.769 & $0.455-0.913$ \\
\hline \multicolumn{5}{|l|}{$\operatorname{PDSR}\left(\mathrm{s}^{-1}\right)$} \\
\hline Radial & 0.782 & $0.490-0.917$ & 0.840 & $0.609-0.941$ \\
\hline Circumferential & 0.794 & $0.513-0.922$ & 0.773 & $0.468-0.914$ \\
\hline
\end{tabular}

All $\mathrm{P}<0.001$. Cl, confidence interval; ICC, intraclass correlation coefficient; LV, left ventricle; LVEF, LV ejection fraction; LVMMT, LV maximum myocardial thickness; PDSR, peak diastolic strain rate; PS, peak strain; PSSR, peak systolic strain rate.

symmetric ventricles (20). In 1986, Messerli proposed that increased body mass needed higher $\mathrm{CO}$ and intravascular volumes to meet the increased metabolic demands (21). Recently, another study reported that increased CO resulting from a higher BMI was affected by $\mathrm{SV}$ independent of heart rate in young adults (22). In the present study, an elevated hemodynamic state was found in the obesity group, with higher SV and CO, which is concordant with previous studies $(4,19,22)$. In individuals with obesity, a state of chronic volume overload can cause a compensatory increase in cardiac preload. Also, in the present study, BP was higher in the obesity group than the 
HC group, indicating increased LV afterload. Generally, higher $\mathrm{BP}$, even within the normal range, can contribute to myocardial remodeling (6). Our data showed that MAP was moderately correlated with LV chamber size, mass, and wall thickness. Similarly, Alpert et al. reported that cardiac load conditions were associated with LV chamber dilation and hypertrophy (myocardial mass and wall thickness) (18).

\section{Obesity and cardiac dysfunction}

The present study did not find any significant differences in LVEF between adults with uncomplicated obesity and HCs, which is consistent with results of previous reports $(4,5,23)$. However, impaired LV contractility was detected during strain analysis, with global radial, circumferential, and longitudinal PS being lower in the obesity group than the HC group. These results indicate that PS has higher sensitivity than LVEF as marker for subclinical myocardial contractile dysfunction. Similar results were reported by Homsi et al. (23), who reported that their obesity group had lower mean LV longitudinal and circumferential strain on CMR than their non-obesity group. However, previous echocardiographic studies revealed decreased longitudinal strain, increased or decreased radial strain, and reduced or no marked changes in circumferential strain among children with obesity $(24,25)$. The apparent inconsistencies between earlier studies and the present study may be attributable to differences in the study populations, sample sizes, and the different imaging modalities used. Also, obese individuals had decreased subclinical diastolic function with lower global longitudinal and circumferential PDSR than HCs. In contrast, subclinical systolic function, represented by PSSR, was not significantly different between the two groups. In children, it has also been demonstrated that although circumferential PDSR is decreased in obesity, there are no significant changes in PSSR on CMR imaging (3). Previous studies have also confirmed that obesity is associated with decreased diastolic function, as evidenced by an increase in the half-time of mitral valve pressure, left atrial diameter, and LV end-diastolic pressure, and a reduced deceleration slope with preservation of systolic function $(7,19)$. However, a recent echocardiography study indicated that both diastolic function (early diastolic strain rate) and systolic function (global systolic longitudinal strain rate) were decreased in obese adolescents who were either metabolically healthy or unhealthy (BMI $35-40 \mathrm{~kg} / \mathrm{m}^{2}$ ) (26). The differences between that echocardiographic study and the present study are that our study population was older and had a lower BMI, and we also used a different imaging modality (CMR) to scan the patients. Together, the findings reported above indicate that cardiac dysfunction caused by mild to moderate obesity in adults can lead to impaired diastolic function.

In this study, impaired LV contractility, as evidenced by reduced radial and longitudinal PS, was negatively correlated with LV myocardial remodeling parameters, including cardiac cavity size and hypertrophy (myocardial mass and myocardial thickness). Previous studies have also shown that myocardial dysfunction is caused by an increased LV load and hypertrophy $(7,17,18,27,28)$. Diffuse myocardial fibrosis observed on $\mathrm{T}_{1}$-weighted mapping was also found to have adverse effects on ventricular function $(23,29)$. In the present study, none of the participants in the obesity group had LGE in the myocardial LV. However, quantitative assessment of ventricular fibrosis using $\mathrm{T}_{1^{-}}$ weighted mapping CMR is more sensitive compared with LGE (30); thus, we could not determine whether a fibrotic change of the $\mathrm{LV}$ myocardium was the main factor in the present study. Moreover, the EAT volume and area were greater in the obesity group than the HC group. EAT located within the pericardium was recognized as VAT with a putative paracrine function that directly affects the myocardium through the uptake and release of free fatty acids and the release of adipokines and cytokines, and sharing the same coronary microcirculation. Previous studies have shown that increased EAT is associated with lipotoxicity, myocardial steatosis, and interstitial myocardial fibrosis, and thereby affects cardiac structure and function $(27,29)$.

Subclinical LV dysfunction in individuals with obesity may also be explained by insulin resistance. In this study, insulin resistance was greater with increased BMI, and was associated with circumferential PS and PDSR even after adjustment for age, sex, height, and MAP. A study of sucrose-fed rats suggested that insulin resistance directly induced cardiac contractility impairment (31), whereas a clinical study in women showed that the link between insulin resistance and myocardial fatty acid metabolism could reduce myocardial contractile function in obesity (32).

\section{Associations between adiposity distribution and cardiac remodeling and dysfunction}

$\mathrm{BMI}$ is used as a measure of general adiposity; however, there are limitations to its use in evaluating the effects of 
obesity on specific organs (6). Therefore, in this study, we also analyzed the correlations of various parameters of adiposity with cardiac remodeling and dysfunction in the obesity group. These analyses showed that BMI was independently associated with myocardial thickness in adults with obesity, even after adjusting for age, sex, MAP, and HOMA-IR. This finding is consistent with that of a CMR obesity study conducted in children (3). Among the adiposity indices, VAT was independently associated with LV subclinical dysfunction, represented by global longitudinal PS, as well as longitudinal and circumferential PDSR. This observation is consistent with one in a previous report, in which VAT was demonstrated to be a better predictor of LV diastolic cardiac function than BMI (4). The results suggest that VAT may be a better indicator of LV subclinical dysfunction in individuals with obesity than BMI, and it may be an important indicator in both determining initial treatment strategies and evaluating the efficacy of treatment. The present findings may be explained by the fact that VAT is a metabolically active tissue that can affect cardiac morphology and function by secreting various cytokines and chemokines (33).

\section{Study limitations}

This study has several limitations. First, the study was a cross-sectional in design. It is still unclear whether LV remodeling and subtle contractile impairment can cause heart failure with the progression of obesity, and whether these changes can be reversed. Therefore, longitudinal studies to explore dynamic cardiac changes and factors potentially involved in the reversal of these changes in individuals with obesity are needed in future. Second, previous studies have reported that various key inflammatory markers and insulin resistance are consistently associated with obesity and obesity-related comorbidities (34-38). In the present study, FINS was not measured in 3 of the 48 participants in the obesity group and in none of the HCs; consequently, these individuals were not included in the HOMA-IR analysis. Moreover, because of data incompleteness, we could not include or analyze inflammatory markers in the present study. Third, although this study evaluated myocardial fibrosis based on LGE images, quantitative $T_{1}$-weighted mapping values are more sensitive indicators of interstitial fibrosis. In future studies, these values should be measured to gain an understanding of the mechanisms underlying myocardial damage in obesity.

\section{Conclusions}

Extensive LV geometric remodeling and marked changes in cardiac strains were observed in adults with obesity in the absence of clinically apparent cardiovascular risk factors. Tissue tracking with CMR revealed subclinical impairment of ventricular function with preserved LVEF in these individuals. BMI was found to be independently related to LV remodeling in individuals with obesity. Finally, HOMAIR and VAT were found to be potentially superior to BMI as predictors of subclinical dysfunction, as assessed by strain, in obesity.

\section{Acknowledgments}

Funding: This work was supported by grants from the National Natural Science Foundation of China (81601462), the Key Research \& Development Project of Science and Technology of Sichuan Province (2021YFS0142), the Science and Technology Department of Sichuan Province (2019YFS0302), and the 1.3.5. Project for Disciplines of Excellence, West China Hospital, Sichuan University (ZYGD18017).

\section{Footnote}

Reporting Checklist: The authors have completed the MDAR checklist. Available at https://dx.doi.org/10.21037/qims-21-724

Conflicts of Interest: All authors have completed the ICMJE uniform disclosure form (available at https://dx.doi. org/10.21037/qims-21-724). XZ is employed by Siemens Healthineers Ltd. The other authors have no conflicts of interest to declare.

Ethical Statement: The authors are accountable for all aspects of the work in ensuring that questions related to the accuracy or integrity of any part of the work are appropriately investigated and resolved. This study was conducted in accordance with the Declaration of Helsinki (as revised in 2013). The study was approved by the Institutional Review Board of the West China Hospital in Sichuan University, and informed consent was obtained from all participants.

Open Access Statement: This is an Open Access article distributed in accordance with the Creative Commons Attribution-NonCommercial-NoDerivs 4.0 International 
License (CC BY-NC-ND 4.0), which permits the noncommercial replication and distribution of the article with the strict proviso that no changes or edits are made and the original work is properly cited (including links to both the formal publication through the relevant DOI and the license). See: https://creativecommons.org/licenses/by-nc-nd/4.0/.

\section{References}

1. Tian Y, Jiang C, Wang M, Cai R, Zhang Y, He Z, et al. BMI, leisure-time physical activity, and physical fitness in adults in China: results from a series of national surveys, 2000-14. Lancet Diabetes Endocrinol 2016;4:487-97.

2. Kenchaiah S, Evans JC, Levy D, Wilson PW, Benjamin EJ, Larson MG, Kannel WB, Vasan RS. Obesity and the risk of heart failure. N Engl J Med 2002;347:305-13.

3. Jing L, Binkley CM, Suever JD, Umasankar N, Haggerty CM, Rich J, Wehner GJ, Hamlet SM, Powell DK, Radulescu A, Kirchner HL, Epstein FH, Fornwalt BK. Cardiac remodeling and dysfunction in childhood obesity: a cardiovascular magnetic resonance study. J Cardiovasc Magn Reson 2016;18:28.

4. Zhu L, Gu S, Wang Q, Zhou X, Wang S, Fu C, Yang W, Wetzl J, Yan F. Left ventricular myocardial deformation: a study on diastolic function in the Chinese male population and its relationship with fat distribution. Quant Imaging Med Surg 2020;10:634-45.

5. Orhan AL, Uslu N, Dayi SU, Nurkalem Z, Uzun F, Erer HB, Hasdemir H, Emre A, Karakus G, Soran O, Gorcsan $\mathrm{J} 3 \mathrm{rd}$, Eren M. Effects of isolated obesity on left and right ventricular function: a tissue Doppler and strain rate imaging study. Echocardiography 2010;27:236-43.

6. Turkbey EB, McClelland RL, Kronmal RA, Burke GL, Bild DE, Tracy RP, Arai AE, Lima JA, Bluemke DA. The impact of obesity on the left ventricle: the Multi-Ethnic Study of Atherosclerosis (MESA). JACC Cardiovasc Imaging 2010;3:266-74.

7. Pascual M, Pascual DA, Soria F, Vicente T, Hernández AM, Tébar FJ, Valdés M. Effects of isolated obesity on systolic and diastolic left ventricular function. Heart 2003;89:1152-6.

8. Iacobellis G, Ribaudo MC, Leto G, Zappaterreno A, Vecci E, Di Mario U, Leonetti F. Influence of excess fat on cardiac morphology and function: study in uncomplicated obesity. Obes Res 2002;10:767-73.

9. Chuang ML, Danias PG, Riley MF, Hibberd MG, Manning WJ, Douglas PS. Effect of increased body mass index on accuracy of two-dimensional echocardiography for measurement of left ventricular volume, ejection fraction, and mass. Am J Cardiol 2001;87:371-4, A10.

10. Grothues F, Smith GC, Moon JC, Bellenger NG, Collins P, Klein HU, Pennell DJ. Comparison of interstudy reproducibility of cardiovascular magnetic resonance with two-dimensional echocardiography in normal subjects and in patients with heart failure or left ventricular hypertrophy. Am J Cardiol 2002;90:29-34.

11. Claus P, Omar AMS, Pedrizzetti G, Sengupta PP, Nagel E. Tissue Tracking Technology for Assessing Cardiac Mechanics: Principles, Normal Values, and Clinical Applications. JACC Cardiovasc Imaging 2015;8:1444-60.

12. Lee JY, Choi JW, Kim H. Determination of body surface area and formulas to estimate body surface area using the alginate method. J Physiol Anthropol 2008;27:71-82.

13. de Simone G, Daniels SR, Devereux RB, Meyer RA, Roman MJ, de Divitiis O, Alderman MH. Left ventricular mass and body size in normotensive children and adults: assessment of allometric relations and impact of overweight. J Am Coll Cardiol 1992;20:1251-60.

14. Prasad M, Ramesh A, Kavanagh P, Gerlach J, Germano G, Berman D, Slomka P. Myocardial wall thickening from gated Magnetic Resonance images using Laplace's equation. Proc SPIE Int Soc Opt Eng 2009.

15. Scatteia A, Baritussio A, Bucciarelli-Ducci C. Strain imaging using cardiac magnetic resonance. Heart Fail Rev 2017;22:465-76.

16. Cohn JN, Ferrari R, Sharpe N. Cardiac remodeling-concepts and clinical implications: a consensus paper from an international forum on cardiac remodeling. Behalf of an International Forum on Cardiac Remodeling. J Am Coll Cardiol 2000;35:569-82.

17. Peterson LR, Waggoner AD, Schechtman KB, Meyer T, Gropler RJ, Barzilai B, Dávila-Román VG. Alterations in left ventricular structure and function in young healthy obese women: assessment by echocardiography and tissue Doppler imaging. J Am Coll Cardiol 2004;43:1399-404.

18. Alpert MA, Lambert CR, Terry BE, Cohen MV, Mukerji V, Massey CV, Hashimi MW, Panayiotou H. Interrelationship of left ventricular mass, systolic function and diastolic filling in normotensive morbidly obese patients. Int J Obes Relat Metab Disord 1995;19:550-7.

19. Powell BD, Redfield MM, Bybee KA, Freeman WK, Rihal CS. Association of obesity with left ventricular remodeling and diastolic dysfunction in patients without coronary artery disease. Am J Cardiol 2006;98:116-20.

20. Bellenger NG, Burgess MI, Ray SG, Lahiri A, Coats AJ, Cleland JG, Pennell DJ. Comparison of left 
ventricular ejection fraction and volumes in heart failure by echocardiography, radionuclide ventriculography and cardiovascular magnetic resonance; are they interchangeable? Eur Heart J 2000;21:1387-96.

21. Messerli FH. Cardiopathy of obesity--a not-so-Victorian disease. N Engl J Med 1986;314:378-80.

22. Wade KH, Chiesa ST, Hughes AD, Chaturvedi N, Charakida M, Rapala A, Muthurangu V, Khan T, Finer N, Sattar N, Howe LD, Fraser A, Lawlor DA, Davey Smith G, Deanfield JE, Timpson NJ. Assessing the causal role of body mass index on cardiovascular health in young adults: Mendelian randomization and recall-by-genotype analyses. Circulation 2018;138:2187-201.

23. Homsi R, Yuecel S, Schlesinger-Irsch U, Meier-Schroers M, Kuetting D, Luetkens J, Sprinkart A, Schild HH, Thomas DK. Epicardial fat, left ventricular strain, and T1relaxation times in obese individuals with a normal ejection fraction. Acta Radiol 2019;60:1251-7.

24. Saltijeral A, Isla LP, Pérez-Rodríguez O, Rueda S, Fernandez-Golfin C, Almeria C, Rodrigo JL, Gorissen W, Rementeria J, Marcos-Alberca P, Macaya C, Zamorano J. Early myocardial deformation changes associated to isolated obesity: a study based on 3D-wall motion tracking analysis. Obesity (Silver Spring) 2011;19:2268-73.

25. Koopman LP, McCrindle BW, Slorach C, Chahal N, Hui W, Sarkola T, Manlhiot C, Jaeggi ET, Bradley TJ, Mertens L. Interaction between myocardial and vascular changes in obese children: a pilot study. J Am Soc Echocardiogr 2012;25:401-410.e1.

26. Siurana JM, Ventura PS, Yeste D, Riaza-Martin L, Arciniegas L, Clemente M, Torres M, Amigó N, Giralt G, Roses-Noguer F, Sabate-Rotes A. Myocardial Geometry and Dysfunction in Morbidly Obese Adolescents (BMI 3540 kg/m²). Am J Cardiol 2021;157:128-34.

27. von Bibra H, St John Sutton M. Diastolic dysfunction in diabetes and the metabolic syndrome: promising potential for diagnosis and prognosis. Diabetologia 2010;53:1033-45.

28. Ohtani T, Mohammed SF, Yamamoto K, Dunlay SM, Weston SA, Sakata Y, Rodeheffer RJ, Roger VL, Redfield MM. Diastolic stiffness as assessed by diastolic wall strain is associated with adverse remodelling and poor outcomes in heart failure with preserved ejection fraction. Eur Heart J 2012;33:1742-9.

29. Ng ACT, Strudwick M, van der Geest RJ, Ng ACC, Gillinder L, Goo SY, Cowin G, Delgado V, Wang WYS, Bax JJ. Impact of Epicardial Adipose Tissue, Left
Ventricular Myocardial Fat Content, and Interstitial Fibrosis on Myocardial Contractile Function. Circ Cardiovasc Imaging 2018;11:e007372.

30. Dass S, Suttie JJ, Piechnik SK, Ferreira VM, Holloway CJ, Banerjee R, Mahmod M, Cochlin L, Karamitsos TD, Robson MD, Watkins H, Neubauer S. Myocardial tissue characterization using magnetic resonance noncontrast $\mathrm{t} 1$ mapping in hypertrophic and dilated cardiomyopathy. Circ Cardiovasc Imaging 2012;5:726-33.

31. Hintz KK, Aberle NS, Ren J. Insulin resistance induces hyperleptinemia, cardiac contractile dysfunction but not cardiac leptin resistance in ventricular myocytes. Int J Obes Relat Metab Disord 2003;27:1196-203.

32. Peterson LR, Herrero P, Schechtman KB, Racette SB, Waggoner AD, Kisrieva-Ware Z, Dence C, Klein S, Marsala J, Meyer T, Gropler RJ. Effect of obesity and insulin resistance on myocardial substrate metabolism and efficiency in young women. Circulation 2004;109:2191-6.

33. Fontana L, Eagon JC, Trujillo ME, Scherer PE, Klein S. Visceral fat adipokine secretion is associated with systemic inflammation in obese humans. Diabetes 2007;56:1010-3.

34. Cox AJ, West NP, Cripps AW. Obesity, inflammation, and the gut microbiota. Lancet Diabetes Endocrinol 2015;3:207-15.

35. Quilliot D, Alla F, Böhme P, Bruntz JF, Hammadi M, Dousset B, Ziegler O, Zannad F. Myocardial collagen turnover in normotensive obese patients: relation to insulin resistance. Int J Obes (Lond) 2005;29:1321-8.

36. Abel ED, Litwin SE, Sweeney G. Cardiac remodeling in obesity. Physiol Rev 2008;88:389-419.

37. Sang T, Guo C, Guo D, Wu J, Wang Y, Wang Y, Chen J, Chen C, Wu K, Na K, Li K, Fang L, Guo C, Wang X. Suppression of obesity and inflammation by polysaccharide from sporoderm-broken spore of Ganoderma lucidum via gut microbiota regulation. Carbohydr Polym 2021;256:117594.

38. Wong CY, O'Moore-Sullivan T, Leano R, Byrne N, Beller E, Marwick TH. Alterations of left ventricular myocardial characteristics associated with obesity. Circulation 2004;110:3081-7.

Cite this article as: Liu $\mathrm{J}, \mathrm{Li} \mathrm{J}, \mathrm{Pu} \mathrm{H}, \mathrm{He} W$, Zhou X, Tong N, Peng L. Cardiac remodeling and subclinical left ventricular dysfunction in adults with uncomplicated obesity: a cardiovascular magnetic resonance study. Quant Imaging Med Surg 2022;12(3):2035-2050. doi: 10.21037/qims-21-724 